\title{
A dietary and lifestyle worksite intervention to reduce high prevalence of overweight and obesity for London firefighters
}

\author{
G.R. Lessons and D. Bhakta \\ School of Human Sciences, Health Sciences, London Metropolitan University, London, N7 8DB, UK.
}

This abstract was awarded the student prize.

Overweight and obesity in UK firefighters are reaching epidemic proportions exceeding that of the UK general population ${ }^{(1)}$. This compromises physical fitness ${ }^{(2)}$ and concomitant firefighter and public safety ${ }^{(3)}$. Research has identified specific exposures related to firefighting ${ }^{(4)}$, which may also contribute to uniquely high rates of coronary heart disease (CHD) and acute myocardial infarctions (MI) seen in this occupational group ${ }^{(6)}$. These exposures include intense heat which could impair vascular function and increase thrombogenicity ${ }^{(4)}$, and fire stations as obesogenic environments which perpetuate a culture of over-nutrition alongside increasing sedentary behaviour ${ }^{(5)}$. Faced with a multitude of exposures, it is imperative that modifiable risk factors for CHD and MI are addressed. This however seems to have been overlooked by UK researchers thus far. We aimed to design, implement and evaluate the efficacy and feasibility of a fire station based dietary and lifestyle weight loss intervention for UK firefighters, factoring in a complex combination of exposures and firefighter specific cultural habits ${ }^{(5)}$.

Forty London firefighters were recruited via staff email in February 2017. Thirty-eight firefighters (35 males, 3 females; mean age $=44.8, \mathrm{SD}=7 \cdot 2$ ) from 2 London fire stations (Hornchurch: intervention, Harold Hill: control) completed the one-month, clustercontrolled dietary and lifestyle intervention which ran February to March 2017. Hornchurch firefighters received the programme involving a face-to-face personalised information session; general information sheet; midway face-to-face support session; and nutritionist support throughout. Informational components focussed on fire station obesogenic environmental modification alongside nutrition, physical activity and lifestyle education in a low-intensity/low participant burden format. All firefighters were assessed at baseline and post-intervention for Body Mass Index (BMI), Body Fat Percentage (BF\%) and Waist Circumference (WC). Dietary assessment was carried out using the EPIC-Norfolk Food Frequency Questionnaire ${ }^{(7)}$. At baseline, $70 \%$ of the sample were classified overweight or obese by BMI (Harold Hill: $67 \%$; Hornchurch: $74 \%$ ). Post intervention, Hornchurch displayed significant mean reductions in $\mathrm{BMI}$ and $\mathrm{BF} \%$ of $0.4 \mathrm{~kg} / \mathrm{m}^{2}(\mathrm{p}=0.01)$ and $0.9 \%(\mathrm{p}=0.04)$ respectively, and borderline non-significant improvement in WC of $1.4 \mathrm{~cm}(\mathrm{p}=0.06)$. Harold Hill displayed a significant mean reduction of $0.8 \mathrm{~cm}$ in WC $(\mathrm{p}=0.03)$, but not for $\mathrm{BMI}$ or $\mathrm{BF} \%$. Hornchurch firefighters reported favourable changes over the one month period for energy intake: $-413 \mathrm{kcal} / \mathrm{d}(\mathrm{p}=0.04)$; saturated fatty acids: $-3.5 \mathrm{~g} / \mathrm{d}(\mathrm{p}=0.01)$; fats $\&$ oils: $-3.2 \mathrm{~g} / \mathrm{d}(\mathrm{p}=0.03)$; sugars, preserves and snacks: $-24 \cdot 1 \mathrm{~g} / \mathrm{d}(\mathrm{p}=0.02)$; cereals and cereal products: $-35.9 \mathrm{~g} / \mathrm{d}(\mathrm{p}=0.04)$.

With an attrition rate of $5 \%$ the intervention was highly feasible. It was also efficacious, resulting in significant weight loss, reduced body fat and improvements in dietary behaviour. This study represents the first dietary and lifestyle worksite intervention for firefighters in the UK.

1. Munir F, Clemes S, Houdmont J et al. (2012) Occup Med 62(5), 362-365.

2. Poston WSC, Haddock CK, Jahnke SA et al. (2011) J Occup Environ Med 53(3), 266-273.

3. Moore AM (2003) J Clin Hypertens 5, 350-351.

4. Hunter AL, Shah ASV, Langrish JP et al. (2017) Circulation 135, 1284-1295.

5. Dobson M, Choi B, Schnall PL et al. (2013) Am J Ind Med 56(7), 776-790.

6. Kales SN, Soteriades ES, Christophi CA et al. (2007) N Engl J Med 356, 1207-1215.

7. Bingham SA, Welch AA, McTaggart A et al. (2001) Public Health Nutr 4(3), 847-858. 